\title{
How Z-DNA/RNA binding proteins shape homeostasis, inflammation, and immunity
}

\author{
Chun Kim $^{*}$ \\ Department of Molecular and Life Science, Hanyang University [ERICA Campus], Ansan 15588, Korea
}

The right-handed double-helical structure of DNA (B-DNA), which follows the Watson-Crick model, is the canonical form of DNA existing in normal physiological settings. Even though an alternative left-handed structure of DNA (Z-DNA) was discovered in the late 1970s, Z-form nucleic acid has not received much attention from biologists, because it is extremely unstable under physiological conditions, has an ill-defined mechanism of its formation, and has obscure biological functions. The debate about the physiological relevance of Z-DNA was settled only after a class of proteins was found to potentially recognize the Z-form architecture of DNA. Interestingly, these Z-DNA binding proteins can bind not only the left-handed form of DNA but also the equivalent structure of RNA (Z-RNA). The Z-DNA/RNA binding proteins present from viruses to humans function as important regulators of biological processes. In particular, the proteins ADAR1 and ZBP1 are currently being extensively re-evaluated in the field to understand potential roles of the noncanonical Z-conformation of nucleic acids in host immune responses and human disease. Despite a growing body of evidence supporting the biological importance of Z-DNA/RNA, there remain many unanswered principal questions, such as when Z-form nucleic acids arise and how they signal to downstream pathways. Understanding Z-DNA/RNA and the sensors in different pathophysiological conditions will widen our view on the regulation of immune responses and open a new door of opportunity to develop novel types of immunomodulatory therapeutic possibilities. [BMB Reports 2020; 53(9): 453-457]

\section{INTRODUCTION}

The well-known model of DNA, which was originally proposed by Watson and Crick, is a right-handed double-helical conformation of B-form DNA (B-DNA). Surprisingly, the first single-cry-

*Corresponding author. Tel: +82-31-400-5512; Fax: +82-31-400-5457; E-mail: chunkim@hanyang.ac.kr

https://doi.org/10.5483/BMBRep.2020.53.9.141

Received 22 June 2020

Keywords: ADAR1, Inflammation, Necroptosis, ZBP1, Z-DNA, Z-RNA stal structure study of DNA discovered that DNA adopted an unexpected zigzag shaped left-handed double helix (Z-DNA) instead of the established B-DNA (1). Later, it turned out that a DNA duplex with alternating purine-pyrimidine sequences such as poly $(\mathrm{dG}-\mathrm{dC})$ can stabilize the Z-DNA conformation under high salt concentrations (2). Since Z-DNA formation typically requires molar concentrations of salts, the significance of this structure under physiological conditions and its biological function has remained elusive.

In attempts to search for potential functions of Z-DNA in a biological system, double-stranded RNA-specific adenosine deaminase 1 (ADAR1) was identified as the first protein that contains a Z-DNA-binding domain $(Z \alpha)$ (3). Spectroscopic measurements revealed that when the $Z \alpha$ domain meets GC-repeated DNA, it can convert the B-DNA to Z-DNA and stabilize the left-handed conformation (4). Interestingly, structural studies revealed that Z-DNA binding to the Z $\alpha$ domain is specific not to a DNA sequence, but to the left-handed conformation $(5,6)$. Furthermore, the Z-DNA equivalent structure of RNA (Z-RNA) was found to bind to the $Z \alpha$ as well $(6,7)$. Soon after the $Z \alpha$ domain in ADAR1 was identified, sequence similarity search identified Z-DNA-binding protein 1 (ZBP1; also known as DLM1 and DAI) having similar motifs (8). In addition, a mammalian PKR-like kinase in fish, named PKZ, uses the Z $\alpha$ domain to promote immune responses (9). The Z-DNA/RNA-recognizing proteins exist not only in animals but also in certain viruses. Poxviruses, including vaccinia virus, are known to have the $Z \alpha$ domain bearing E3L protein (10). When the Z $\alpha$ domain of E3L was removed, the infection was attenuated, suggesting that Z-DNA/RNA binding activity is crucial for viral pathogenicity $(10,11)$. Taken together, the identification of a class of proteins that bind specifically to Z-DNA/RNA suggested that noncanonical Z-form nucleic acids can have biological functions in vivo. In this mini-review, I discuss the recent advances in understanding the potential role of Z-form nucleic acid-recognizing proteins in cell death, inflammation, and immunity.

\section{ADAR1}

The ADAR1 gene expresses two isoforms of adenosine deaminases by taking advantage of its alternative promoters and start codons (12-14). The constitutively expressed short isoform, ADAR1p110, is mainly located in the nucleus, whereas the

ISSN: 1976-670X (electronic edition)

Copyright (C) 2020 by the The Korean Society for Biochemistry and Molecular Biology

(ㄷ) This is an open-access article distributed under the terms of the Creative Commons Attribution Non-Commercial License (http://creativecommons.org/licenses/by-nc/4.0) which permits unrestricted non-commercial use, distribution, and reproduction in any medium, provided the original work is properly cited. 


\begin{tabular}{|c|c|c|c|c|c|c|c|}
\hline ZBP1 (DAI, DLM1) & $Z \alpha$ & $\mathrm{Za}$ & RHIM & RHIM & & & \\
\hline ADAR1 (p150) & & $\mathrm{Z} \alpha$ & $\mathrm{Z} \beta$ & RBD & RBD & RBD & Deaminase \\
\hline ADAR1 (p110) & & & $Z \beta$ & RBD & RBD & RBD & Deaminase \\
\hline E3L & $\mathrm{Z} \alpha$ & RBD & & & & & \\
\hline PKZ & $Z \alpha$ & $Z \alpha$ & & Kinase & & & \\
\hline
\end{tabular}

Fig. 1. Functional Domains of Z-DNA/RNA binding proteins. The two Z $\alpha$ domain-containing ZBP1 establishes cell death by interaction with RIPK1 and RIPK3 via its RHIM domains. The ADAR1p150 isoform comprises a Z $\alpha$ domain, a nonfunctional Z $\beta$, three RBD (dsRNA binding domain), and a deaminase domain that edits dsRNA. E3L from vaccinia virus has one Z $\alpha$ domain required for viral pathogenesis. PKZ is a fish kinase-recognizing Z-nucleic acid. The physiological role of Z $\alpha$ in all these Z-DNA/RNA sensing proteins is still largely unknown.

cytoplasm-predominant long isoform, ADAR1p150, is interferon (IFN)-inducible and shuttles between the nucleus and the cytoplasm $(15,16)$. Both ADAR1p110 and p150 isoforms share the deaminase domain, double-strand RNA-binding domains (RBD), and a putative Z-DNA-binding domain, $Z \beta$. The isoform P150 has an extra Z-DNA/RNA-binding domain, Z $\alpha$ at its N-terminal end (Fig. 1) (17). Of note, despite having a similar amino-acid sequence and structural architecture with $Z \alpha, Z \beta$ appears not to recognize Z-form nucleic acids (18). Currently, the function of $Z \beta$ is unclear.

A purine nucleoside, inosine, can be generated by deamination of adenosine. ADAR1 deaminates adenosine (A) to inosine (I) in dsRNA species, by employing its RBD and deaminase domain. Most of the A-to-I RNA editing by ADAR1 occurs within Alu elements embedded within mRNA (19). Mutations in the ADAR1 gene are implicated in Aicardi-Goutières syndrome (AGS), a fatal childhood autoimmune disease characterized by type I interferonopathies (20). Similar to human AGS, Adar1 KO and Adar1 deaminase-inactive mutant mice display embryonic lethality with high levels of expression of interferon-stimulated genes (ISG) (21-24). One of the antiviral defense pathways inducing IFN is a non-self dsRNA-recognition system that uses a sensor, MDA5, and an adaptor, MAVS, proteins. Ablation of MDA5 or MAVS rescues the embryonic lethality and the pathogenic type I IFN production in Adar1 $\mathrm{KO}$ and Adar1 deaminase-inactive mutant animals $(24,25)$. Therefore, ADAR1-mediated RNA editing prevents MDA5-dependent recognition of self RNA and the following signal transduction to MAVS, suppressing uncontrolled IFN production (Fig. 2).

ADAR1p150-specific mutations are found in patients with dyschromatosis symmetrica hereditarian (DSH), which is characterized by irregular skin pigmentation (26). In contrast to mostly autosomal recessive AGS, DSH is an autosomal dominant disease. The mutations located on the upstream of the initiation codon of $\mathrm{p} 110$ generate early stop codons in ADAR $1 \mathrm{p} 150$, but the expression of $\mathrm{p} 110$ is unaffected. DSH pathology seems to be determined by the insufficient level of $Z \alpha-$ containing ADAR1p150. In this regard, although ADAR1 has been studied mainly for its RBD and deaminase-mediated RNA editing, one can speculate that there is a Z $\alpha$-specific

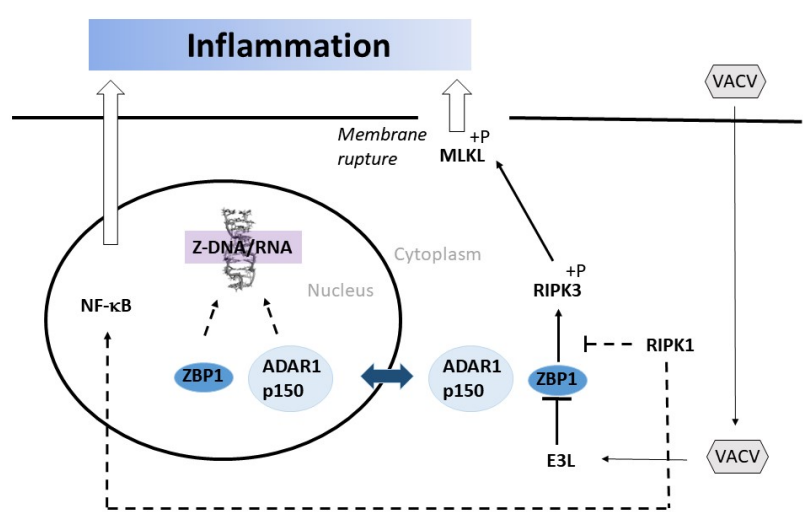

Fig. 2. Z-nucleic acid-dependent inflammatory signaling pathway. ADAR1p150 and ZBP1 seem to recognize stress-induced Z-conformational nucleic acids. Although it is not experimentally demonstrated yet, the potential interplay between ADAR1p150 and ZBP1 is expected since both proteins are interferon-inducible and equally recognize Z-DNA/RNA through their $Z \alpha$ domains. The double-headed arrow indicates the nucleocytoplasmic shuttling of ADAR1p150 and ZBP1. While Z-nucleic acid-triggered ZBP1-dependent cell death pathway is currently actively being investigated, Z-DNARNA-dependent function of ADAR1p150 is still largely unknown. Some viruses such as vaccinia virus (VACV) can prevent host Z-DNA/RNA sensing by their Z $\alpha$ domain-bearing E3L protein. The mechanisms of signaling pathways with dashed lines are less well-established. Even though RIPK1-dependent NF- $\kappa B$ activation is well-known, how Z-DNA/RNA-dependent ZBP1 complex signals to RIPK1 remains elusive.

function of ADAR1. Unfortunately, the role of the Z $\alpha$ domain in ADAR1p150 is currently ill-defined. One study suggests that $Z \alpha$ improves the RNA editing efficiency of ADAR1 when the target dsRNA has a Z-conformation-prone sequence proximal to A-to-I editing sequences (27). Also, the $Z \alpha$ domain was required for the localization to stress granules (SG), which is a transient cytoplasmic aggregate generated in response to translation-arresting cellular stresses (28). Since SG is mainly composed of mRNPs and certain RNA binding proteins, SG can interfere with viral replication by stopping the host translation machinery (29). Even though the physiological meaning 
of Z $\alpha$-dependent localization to SG is unclear yet, SG-located ADAR1 may help resolve the aggregate by editing RNA duplexes produced by interactions between mRNAs (30).

\section{ZBP1}

Z-DNA binding protein 1 (ZBP1) was initially found as a highly expressed gene in tumor stroma (31). Although one study proposed ZBP1 as a cytoplasmic DNA receptor activating type I interferon (IFN) and NF- $\mathrm{BB}$ signalling pathways, subsequent studies demonstrated that ZBP1 was dispensable for cytosolic DNA-mediated host IFN responses (32-34). ZBP1 contains two $\mathrm{N}$-terminal Z $\alpha$ domains, two RIP homotypic interaction motifs (RHIM), and one potential RHIM-like motif with a less-conserved core RHIM sequence (35). The RHIM provides a crucial interaction platform to establish necroptosis, which is a new form of caspase-independent programmed cell death (Fig. 2) (36). In mammals, RHIM is found in four proteins, Receptor Interacting Protein Kinase 1 (RIPK1), RIPK3, TIR-domain-containing adapter-inducing interferon- $\beta$ (TRIF), and ZBP1 (37, 38). In contrast to apoptosis that does not result in inflammation, necroptosis is implicated in pathological inflammatory conditions. Upon necroptotic stimuli, particularly under conditions in which caspase-8 is inactive, RHIM-dependent protein interactions promote RIPK3-dependent phosphorylation of pseudokinase, Mixed Lineage Kinase Like (MLKL). The modification of MLKL drives its oligomerization, licensing MLKL to permeabilize cellular membranes (36).

Although Z-DNA and Z-RNA are potential ligands of ZBP1, it remains unknown where, when, and how Z-form nucleic acids are generated to activate ZBP1-dependent cellular responses. Nevertheless, ZBP1 was shown to be triggered in response to several viral infections, including murine cytomegalovirus (MCMV), herpes simplex viruses (HSV) 1, and influenza A virus (IAV) (39-43). Furthermore, two independent studies demonstrated that in RIPK1 KO and RIPK1 RHIM mutant animals, ZBP1 serves as a potent inducer of necroptosis in response to unidentified endogenous cellular stress $(44,45)$. Taken together, ZBP1 protein proposes the existence of a Z-DNA/RNA-dependent signalling pathway that is important for anti-viral immunity, tissue homeostasis, and inflammation.

Current understanding of ZBP1 is limited to its function of inducing cell death. Intriguingly, an unbiased screening identified ZBP1 as one of the most inhibitory proteins against multiple + ssRNA virus infections (46). Since the screening was done in necroptosis-resistant Hela cells, the result indicates that ZBP1 has unidentified anti-viral functions apart from its role in necroptosis. Indeed, a recent study revealed that zika virus infection-activated ZBP1 restricted viral replication by changing cellular metabolism rather than by inducing necroptosis (47). Furthermore, some studies demonstrated that overexpression of ZBP1 boosted effective cytotoxic T lymphocytes and longlasting antitumor immunity in vivo, suggesting its potential role in host-adaptive immunity $(48,49)$.

\section{FUTURE PERSPECTIVES}

Though known for decades, our understanding about the relationship between Z-DNA/RNA and the sensors ADAR1 and ZBP1 is largely limited to their biochemical aspects. The physiological origin of Z-form nucleic acids and the pathological effects of sensing Z-DNA/RNA remain still enigmatic. Since both ADAR1 and ZBP1 are interferon-inducible proteins, the potential cross talk between these two proteins in sensing Z-DNA/RNA is highly anticipated. The two proteins may compete for occupying Z-nucleic acids, or they may exhibit synergistic effects to establish inflammatory signaling pathways.

While I was preparing this manuscript, two studies reported that endogenous retroelements-derived dsRNA may serve as the agonist of ZBP1 in specific mouse models of skin and intestinal inflammation $(50,51)$. Nonetheless, since experimental data supporting that retroelements are direct targets of the $Z \alpha$ domain are lacking in these studies, other possibilities should remain valid. In fact, another recent study showed that in the mouse prefrontal cortex, the Z $\alpha$ domain of ADAR1 recognizes Z-DNA in host genomes to control gene expression during fear learning (52).

An emerging body of evidence proposes that there is a largely unexplored Z-DNA/RNA-activated signal transduction pathway that plays a role in host immunity, tissue homeostasis, inflammation, and cancer. We are still at the beginning of searching for the origin of Z-form nucleic acids and their potential functions in various physiological conditions. Understanding the biological roles of Z-DNA/RNA and the sensors ADAR1 and ZBP1 is fundamental to fully leverage their therapeutic potentials.

\section{ACKNOWLEDGEMENTS}

This work was supported by the research fund of Hanyang University (HY-2018-N) and the National Research Foundation of Korea (NRF) grant funded by the Korean government (MSIT) (No. 2020R1F1A1074601).

\section{CONFLICTS OF INTEREST}

The author has no conflicting interests.

\section{REFERENCES}

1. Wang AH, Quigley GJ, Kolpak FJ et al (1979) Molecular structure of a left-handed double helical DNA fragment at atomic resolution. Nature 282, 680-686

2. Drew H, Takano T, Tanaka S, Itakura K and Dickerson RE (1980) High-salt d(CpGpCpG), a left-handed Z' DNA double helix. Nature 286, 567-573

3. Herbert A, Lowenhaupt K, Spitzner J and Rich A (1995) Chicken double-stranded RNA adenosine deaminase has apparent specificity for Z-DNA. Proc Natl Acad Sci U S A 92, 7550-7554 
4. Berger I, Winston W, Manoharan R et al (1998) Spectroscopic characterization of a DNA-binding domain, Z alpha, from the editing enzyme, dsRNA adenosine deaminase: evidence for left-handed Z-DNA in the Z alpha-DNA complex. Biochemistry 37, 13313-13321

5. Schwartz T, Rould MA, Lowenhaupt K, Herbert $A$ and Rich A (1999) Crystal structure of the Zalpha domain of the human editing enzyme ADAR1 bound to left-handed Z-DNA. Science 284, 1841-1845

6. Placido D, Brown BA 2nd, Lowenhaupt K, Rich A and Athanasiadis A (2007) A left-handed RNA double helix bound by the $\mathrm{Z}$ alpha domain of the RNA-editing enzyme ADAR1. Structure 15, 395-404

7. Brown BA 2nd, Lowenhaupt K, Wilbert CM, Hanlon EB and Rich A (2000) The zalpha domain of the editing enzyme dsRNA adenosine deaminase binds left-handed Z-RNA as well as Z-DNA. Proc Natl Acad Sci U S A 97, 13532-13536

8. Schwartz T, Behlke J, Lowenhaupt $K$, Heinemann $U$ and Rich A (2001) Structure of the DLM-1-Z-DNA complex reveals a conserved family of Z-DNA-binding proteins. Nat Struct Biol 8, 761-765

9. Rothenburg S, Deigendesch N, Dittmar K et al (2005) A PKR-like eukaryotic initiation factor 2alpha kinase from zebrafish contains Z-DNA binding domains instead of dsRNA binding domains. Proc Natl Acad Sci U S A 102, 1602-1607

10. Kim YG, Muralinath M, Brandt T et al (2003) A role for Z-DNA binding in vaccinia virus pathogenesis. Proc Natl Acad Sci U S A 100, 6974-6979

11. Koehler H, Cotsmire S, Langland J et al (2017) Inhibition of DAl-dependent necroptosis by the Z-DNA binding domain of the vaccinia virus innate immune evasion protein, E3. Proc Natl Acad Sci U S A 114, 11506-11511

12. Kim U, Garner TL, Sanford T, Speicher D, Murray JM and Nishikura K (1994) Purification and characterization of double-stranded RNA adenosine deaminase from bovine nuclear extracts. J Biol Chem 269, 13480-13489

13. O'Connell MA, Krause S, Higuchi M et al (1995) Cloning of cDNAs encoding mammalian double-stranded RNA-specific adenosine deaminase. Mol Cell Biol 15, 1389-1397

14. Patterson JB and Samuel CE (1995) Expression and regulation by interferon of a double-stranded-RNA-specific adenosine deaminase from human cells: evidence for two forms of the deaminase. Mol Cell Biol 15, 5376-5388

15. Poulsen $\mathrm{H}$, Nilsson J, Damgaard CK, Egebjerg J and Kjems J (2001) CRM1 mediates the export of ADAR1 through a nuclear export signal within the Z-DNA binding domain. Mol Cell Biol 21, 7862-7871

16. Strehblow A, Hallegger M and Jantsch MF (2002) Nucleocytoplasmic distribution of human RNA-editing enzyme ADAR1 is modulated by double-stranded RNA-binding domains, a leucine-rich export signal, and a putative dimerization domain. Mol Biol Cell 13, 3822-3835

17. Gallo A, Vukic D, Michalik D, O'Connell MA and Keegan LP (2017) ADAR RNA editing in human disease; more to it than meets the I. Hum Genet 136, 1265-1278

18. Athanasiadis A, Placido D, Maas S, Brown BA 2nd, Lowenhaupt K and Rich A (2005) The crystal structure of the Zbeta domain of the RNA-editing enzyme ADAR1 reveals distinct conserved surfaces among Z-domains. J Mol Biol 351, 496-507

19. Chung $H$, Calis JJA, Wu X et al (2018) Human ADAR1 Prevents Endogenous RNA from Triggering Translational Shutdown. Cell 172, 811-824.e14

20. Rice GI, Kasher PR, Forte GM et al (2012) Mutations in ADAR1 cause Aicardi-Goutieres syndrome associated with a type I interferon signature. Nat Genet 44, 1243-1248

21. Wang Q, Khillan J, Gadue P and Nishikura K (2000) Requirement of the RNA editing deaminase ADAR1 gene for embryonic erythropoiesis. Science 290, 1765-1768

22. Hartner JC, Schmittwolf C, Kispert A, Muller AM, Higuchi $M$ and Seeburg PH (2004) Liver disintegration in the mouse embryo caused by deficiency in the RNA-editing enzyme ADAR1. J Biol Chem 279, 4894-4902

23. Wang Q, Miyakoda M, Yang W et al (2004) Stress-induced apoptosis associated with null mutation of ADAR1 RNA editing deaminase gene. J Biol Chem 279, 4952-4961

24. Liddicoat BJ, Piskol R, Chalk AM et al (2015) RNA editing by ADAR 1 prevents MDA5 sensing of endogenous dsRNA as nonself. Science 349, 1115-1120

25. Mannion NM, Greenwood SM, Young R et al (2014) The RNA-editing enzyme ADAR1 controls innate immune responses to RNA. Cell Rep 9, 1482-1494

26. Suzuki N, Suzuki T, Inagaki K et al (2005) Mutation analysis of the ADAR1 gene in dyschromatosis symmetrica hereditaria and genetic differentiation from both dyschromatosis universalis hereditaria and acropigmentatio reticularis. J Invest Dermatol 124, 1186-1192

27. Koeris M, Funke L, Shrestha J, Rich A and Maas S (2005) Modulation of ADAR1 editing activity by Z-RNA in vitro. Nucleic Acids Res 33, 5362-5370

28. Ng SK, Weissbach R, Ronson GE and Scadden AD (2013) Proteins that contain a functional Z-DNA-binding domain localize to cytoplasmic stress granules. Nucleic Acids Res 41, 9786-9799

29. Protter DS and Parker R (2016) Principles and Properties of Stress Granules. Trends Cell Biol 26, 668-679

30. Herbert A (2019) Z-DNA and Z-RNA in human disease. Commun Biol 2, 7

31. Fu Y, Comella N, Tognazzi K, Brown LF, Dvorak HF and Kocher O (1999) Cloning of DLM-1, a novel gene that is up-regulated in activated macrophages, using RNA differential display. Gene 240, 157-163

32. Takaoka A, Wang Z, Choi MK et al (2007) DAI (DLM-1/ ZBP1) is a cytosolic DNA sensor and an activator of innate immune response. Nature 448, 501-505

33. Ishii KJ, Kawagoe T, Koyama S et al (2008) TANK-binding kinase-1 delineates innate and adaptive immune responses to DNA vaccines. Nature $451,725-729$

34. Lippmann J, Rothenburg S, Deigendesch N et al (2008) IFNbeta responses induced by intracellular bacteria or cytosolic DNA in different human cells do not require ZBP1 (DLM-1/DAI). Cell Microbiol 10, 2579-2588

35. Kuriakose T and Kanneganti TD (2018) ZBP1: Innate Sensor Regulating Cell Death and Inflammation. Trends Immunol 39, 123-134

36. Pasparakis $M$ and Vandenabeele $P$ (2015) Necroptosis and its role in inflammation. Nature $517,311-320$

37. Kaiser WJ, Upton JW and Mocarski ES (2008) Receptor- 
interacting protein homotypic interaction motif-dependent control of NF-kappa B activation via the DNA-dependent activator of IFN regulatory factors. J Immunol 181, 6427-6434

38. Rebsamen M, Heinz LX, Meylan E et al (2009) DAI/ZBP1 recruits RIP1 and RIP3 through RIP homotypic interaction motifs to activate NF-kappaB. EMBO Rep 10, 916-922

39. Upton JW, Kaiser WJ and Mocarski ES (2012) DAI/ZBP1/ DLM-1 complexes with RIP3 to mediate virus-induced programmed necrosis that is targeted by murine cytomegalovirus vIRA. Cell Host Microbe 11, 290-297

40. Pham TH, Kwon KM, Kim YE, Kim KK and Ahn JH (2013) DNA sensing-independent inhibition of herpes simplex virus 1 replication by DAI/ZBP1. J Virol 87, 3076-3086

41. Kuriakose T, Man SM, Malireddi RK et al (2016) ZBP1/DAI is an innate sensor of influenza virus triggering the NLRP3 inflammasome and programmed cell death pathways. Sci Immunol 1, aag2045

42. Thapa RJ, Ingram JP, Ragan KB et al (2016) DAI Senses Influenza A Virus Genomic RNA and Activates RIPK3-Dependent Cell Death. Cell Host Microbe 20, 674-681

43. Maelfait J, Liverpool L, Bridgeman A, Ragan KB, Upton JW and Rehwinkel J (2017) Sensing of viral and endogenous RNA by ZBP1/DAI induces necroptosis. EMBO J 36, 2529-2543

44. Lin J, Kumari S, Kim C et al (2016) RIPK1 counteracts ZBP1-mediated necroptosis to inhibit inflammation. Nature $540,124-128$

45. Newton K, Wickliffe KE, Maltzman A et al (2016) RIPK1 inhibits ZBP1-driven necroptosis during development. Nature 540, 129-133

46. Schoggins JW, MacDuff DA, Imanaka N et al (2014) Pan-viral specificity of IFN-induced genes reveals new roles for cGAS in innate immunity. Nature 505, 691-695

47. Daniels BP, Kofman SB, Smith JR et al (2019) The Nucleotide Sensor ZBP1 and Kinase RIPK3 Induce the Enzyme IRG1 to Promote an Antiviral Metabolic State in Neurons. Immunity 50, 64-76 e64

48. Lladser A, Mougiakakos D, Tufvesson $\mathrm{H}$ et al (2011) DAI (DLM-1/ZBP1) as a genetic adjuvant for DNA vaccines that promotes effective antitumor CTL immunity. Mol Ther 19, 594-601

49. Hirvinen M, Capasso C, Guse K et al (2016) Expression of DAl by an oncolytic vaccinia virus boosts the immunogenicity of the virus and enhances antitumor immunity. Mol Ther Oncolytics 3, 16002

50. Jiao H, Wachsmuth L, Kumari S et al (2020) Z-nucleic-acid sensing triggers ZBP1-dependent necroptosis and inflammation. Nature 580, 391-395

51. Wang R, Li H, Wu J et al (2020) Gut stem cell necroptosis by genome instability triggers bowel inflammation. Nature 580, 386-390

52. Marshall PR, Zhao Q, Li X et al (2020) Dynamic regulation of Z-DNA in the mouse prefrontal cortex by the RNAediting enzyme Adar1 is required for fear extinction. Nat Neurosci 23, 718-729 